\title{
Analgesic effect of quetiapine in a mouse model of cancer-induced bone pain
}

\author{
Mi Hwa Heo ${ }^{1}$, Jin Young Kim ${ }^{1}$ Ilseon Hwang ${ }^{2}$, Eunyoung $\mathrm{Ha}^{3}$, and Keon Uk Park ${ }^{1,4}$
}

Departments of ${ }^{1}$ Internal Medicine and ${ }^{2}$ Pathology, ${ }^{3}$ Pain Research Center, ${ }^{4}$ Keimyung University Kidney Institute, Keimyung University Dongsan Medical Center, Daegu, Korea

Received: November 23, 2015

Revised : October 6, 2016

Accepted: October 6, 2016

\section{Correspondence to}

Keon Uk Park, M.D.

Department of Internal Medicine, Keimyung University Dongsan Medical Center, 56 Dalseongro, Jung-gu, Daegu 41931, Korea

Tel: +82-53-250-8097

Fax: +82-53-425-6476

E-mail: keonukpark@gmail.com
Background/Aims: Cancer-induced bone pain (CIBP) is one of the most common pains in patients with advanced neoplasms. Because of treatment-associated side effects, more than half of cancer patients are reported to have inadequate and undermanaged pain control. New mechanism-based therapies must be developed to reduce cancer pain. Quetiapine is a commonly used atypical antipsychotic drug. We report a study of the potential analgesic effects of quetiapine in a mouse model of CIBP and examine the mechanism of bone pain by analyzing the expression of various nociceptors.

Methods: Fifteen male $\mathrm{C}_{3} \mathrm{H} / \mathrm{HeN}$ mice were arbitrarily divided into five groups: control and, CIBP with no treatment, quetiapine treatment, opioid treatment, and melatonin treatment. The mice were tested for mechanical hyperalgesia by determining the nociceptive hind paw withdrawal pressure threshold. Tissues from tibia were removed and subjected to quantitative and qualitative evaluations of transient receptor potential vanilloid 1 (TRPV1), TRPV4, acid-sensing ion channel 1 (ASIC1), ASIC2, and $\mathrm{ASIC}_{3}$ expression.

Results: Paw withdrawal pressure threshold was improved in the quetiapine treatment group compared with the CIBP group. Expression of TRPV1, TRPV4, $\mathrm{ASIC} 1, \mathrm{ASIC} 2$, and $\mathrm{ASIC}_{3}$ in the CIBP with quetiapine treatment group was significantly lower than that in the CIBP group.

Conclusions: Our results suggest an analgesic effect of quetiapine in the CIBP animal model and implicate TRPV and ASICs as potential targets for cancer pain management.

Keywords: Quetiapine fumarate; Pain; Neoplasms; Nociceptors; Mice

\section{INTRODUCTION}

Pain is a highly prevalent symptom in cancer patients. Cancer-induced bone pain (CIBP) is one of the most common pains in patients with advanced cancer [1]. The treatment of CIBP involves various approaches including radiotherapy, chemotherapy, and medical treatment with bisphosphonates, nonsteroidal anti-inflammatory drugs, and opioid analgesics [2,3]. It has been reported that more than half of cancer patients have inadequate and undermanaged pain control because of treatment-associated side effects $[4,5]$. Therefore, new mechanism-based therapies are needed to reduce cancer pain. An animal model of cancer pain involving injection of osteolytic sarcoma cells into the intramedullary space of the mouse femur has been developed and shows a correlation between tissue-induced tumor destruction, neurochemical changes in sensory neurons and spinal cord, and the development of pain-related behaviors [6]. Sensory information from peripheral tissues is trans- 
mitted to the spinal cord and brain by primary afferent sensory neurons. Specialized sensory neurons known as nociceptors detect environmental stimuli and convert them into electrochemical signals that are transmitted to the central nervous system. Tumors secrete a variety of factors that sensitize or directly excite primary afferent neurons, causing the sensation of pain. Receptors for many of these factors are expressed by primary afferent neurons. The intracellular and extracellular $\mathrm{pH}$ of solid tumors is lower than that of surrounding normal tissues, which can also activate sensory neurons and cause pain in cancer patients [7]. Two acid-sensing ion channels (ASICs) expressed by nociceptors are transient receptor potential vanilloid 1 (TRPV1) and ASICs [8-10]. These channels are sensitized and excited by a decrease in $\mathrm{pH}$ in the range of 4.0 to 5.0 [11].

Quetiapine is a commonly used atypical antipsychotic drug that has superior therapeutic effects in patients with schizophrenia and other neurologic disorders such as depression [12]. Numerous studies related to the anti-inflammatory effects of antidepressants have been reported [13,14], including a study of anti-inflammatory effect of quetiapine on collagen-induced arthritis in mouse model in our center [15].

In this study, we focus on the potential analgesic effects of quetiapine in a CIBP mouse model and evaluate the mechanism of bone pain by analyzing the expression of various nociceptors.

\section{METHODS}

\section{Animals}

$\mathrm{C}_{3} \mathrm{H} / \mathrm{HeN}$ mice (SLC Inc., Hamamatsu, Japan; 6 weeks old) were housed in polycarbonate cages and fed standard mouse chow (Ralston Purina, St. Louis, MO, USA) and water ad libitum. All experimental procedures were examined and approved by the Animal Research Ethics Committee of Keimyung University (KM 2010-28).

\section{Experimental surgical procedure}

Fifteen male $\mathrm{C}_{3} \mathrm{H} / \mathrm{HeN}$ mice were arbitrarily divided into five groups ( $\mathrm{n}=3$ per group) according to intraperitoneal injection regimen as follows: control group, CIBP group, CIBP with quetiapine treatment, CIBP with opioid treatment, and CIBP with melatonin treatment.
The CIBP model was generated by injection of osteolytic fibrosarcoma cells (cell line: NCTC clone 2472) directly into the tibial bone marrow cavity. Control mice underwent the same surgical procedure of injection with the same volume of saline. Treatments were started when the mice showed positive signs of bone tumor on day 28 after surgery. Quetiapine ( $10 \mathrm{mg} / \mathrm{kg}$ ), fentanyl citrate $(10 \mu \mathrm{g} / \mathrm{kg})$, and melatonin (100 ng/kg) were administered through intraperitoneal injection. The mice were injected with the appropriate drug five times a week for 2 weeks. The control mice and CIBP mice were injected with saline. Blood samples and tibial tissues were collected from all mice at the end of the experimental period and stored at $-70^{\circ} \mathrm{C}$ until use.

\section{Behavior test}

The mice were tested for mechanical hyperalgesia by determining the nociceptive hind paw withdrawal pressure threshold (PWPT) with a Paw Pressure Analgesia Instrument (UgoBasile, Monvalle, Italy). The tests were performed by an experimenter who was blinded to the treatment groups. The mice were gently held in the hand while incremental pressure, measured by using an automated gauge, from a $1.75 \mathrm{~mm}^{2}$, blunt, wedge-shaped piston was applied to the dorsal surface of the hind paw. The end point was paw withdrawal. The minimum paw pressure (in grams) that elicited paw withdrawal was defined as the PWPT. Mean PWPT was established by averaging the values of five consecutive tests, separated by intervals of 30 seconds. The PWPT was tested on days 3 , $7,11,14,24,28,32,36$, and 40 .

\section{Reverse transcription-polymerase chain reaction}

At the end of the treatment period, tissues of the left tibia were removed and subjected to quantitative and qualitative evaluations of TRPV1, TRPV4, ASIC1, ASIC2, and $\mathrm{ASIC}_{3}$ expression. Total RNA of tibial tissue from each experimental group was pooled with Trizol (Gibco, Grand Island, NY, USA) according to the manufacturer's protocol and divided into two samples. For reverse transcription-polymerase chain reaction (RT-PCR), $2 \mu \mathrm{g}$ of total RNA was reverse transcribed for 1 hour at $37^{\circ} \mathrm{C}$ in a reaction mixture containing RNA, 40 units RNase inhibitor (Amersham, Piscataway, NJ, USA), $0.5 \mathrm{mM}$ deoxynucleotide triphosphate (Boehringer Mannheim, Indianapolis, IN, USA), $2 \mu \mathrm{M}$ random hexamer primers 
(Stratagene, La Jolla, CA, USA), $5 \times$ avian myeloblastosis virus (AMV) reverse transcriptase reaction buffer, and 30 units AMV reverse transcriptase (Promega, Madison, WI, USA). PCR was performed three times in duplicate using the cDNA as a template. Levels of TRPV1, TRPV4, $\mathrm{ASIC} 1, \mathrm{ASIC} 2$, and $\mathrm{ASIC}_{3}$ expression were determined by normalizing to glyceraldehyde 3-phosphate dehydrogenase (GAPDH) expression. The primers used for TRPV1, TRPV4, ASIC1, ASIC2, and ASIC3 were as follows: forward, 5'-CTT GCC AAG TTT CCT CTT GC-3'; reverse, 5'-CAC CCT CAA CAC ACG TCA TC-3'.

\section{RESULTS}

\section{Radiologic and pathologic findings of tumor development}

Six weeks after injection with fibrosarcoma cells, the tibia of the CIBP mice showed radiolucent lesions, loss of medullary bone, and destruction of cortical bone (Figs. 1 and 2). Pathologic sections of left tibia from CIBP mice showed that tumor cells were densely packed in the marrow cavity and had induced destruction of trabeculae (Fig. 3).

\section{Mechanical hyperalgesia}

PWPT was decreased significantly in the mice with transplanted cancer cells. The PWPT in the CIBP with no treatment group continued to decrease for 40 days (Fig. 4). In contrast, PWPT was improved in the CIBP with quetiapine treatment group compared with the CIBP group. Therefore, a potential analgesic effect was observed in the quetiapine treatment group.

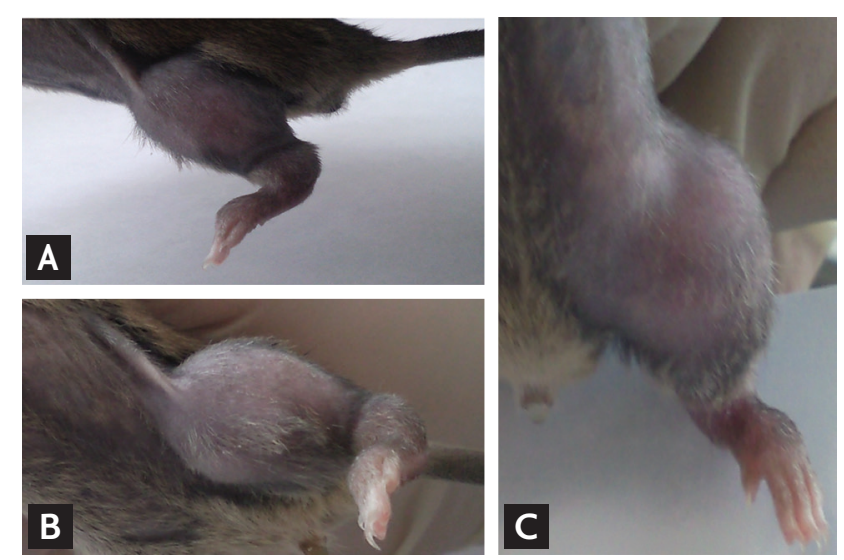

6 Weeks

Figure 1. (A-C) At 6 weeks after injection of tumor cells, the left leg of the cancer-induced bone pain mouse model shows redness and swelling.

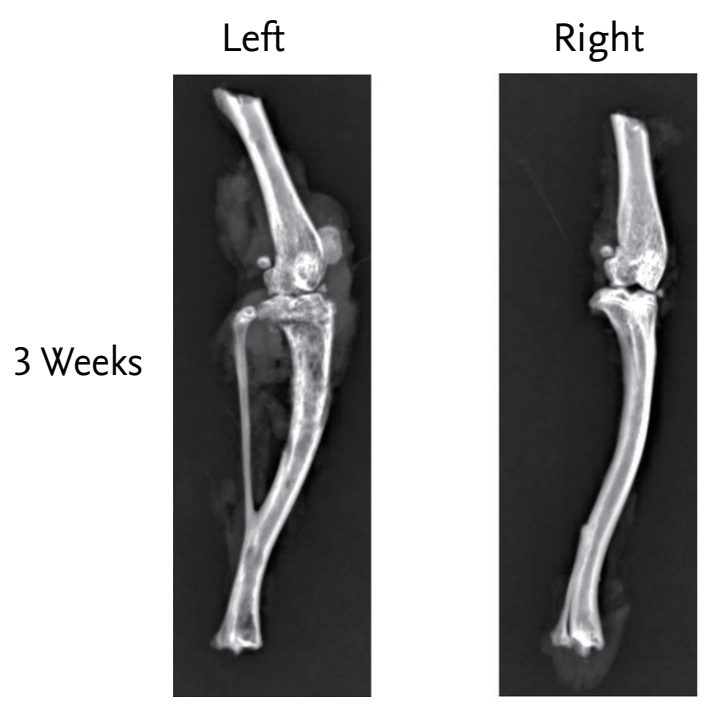

A Tumor cells-injected
Left

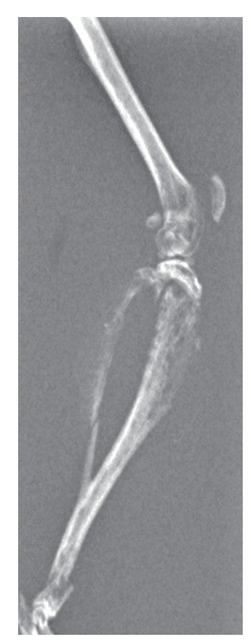

B Tumor cells-injected

Figure 2. Radiologic findings of legs of cancer-induced bone pain (CIBP) mouse model (A) 3 and (B) 6 weeks after injection of tumor cells into the left tibia. X-ray film shows structural destruction of bone marrow of the left leg of the CIBP mouse model compared with the right tibia. 

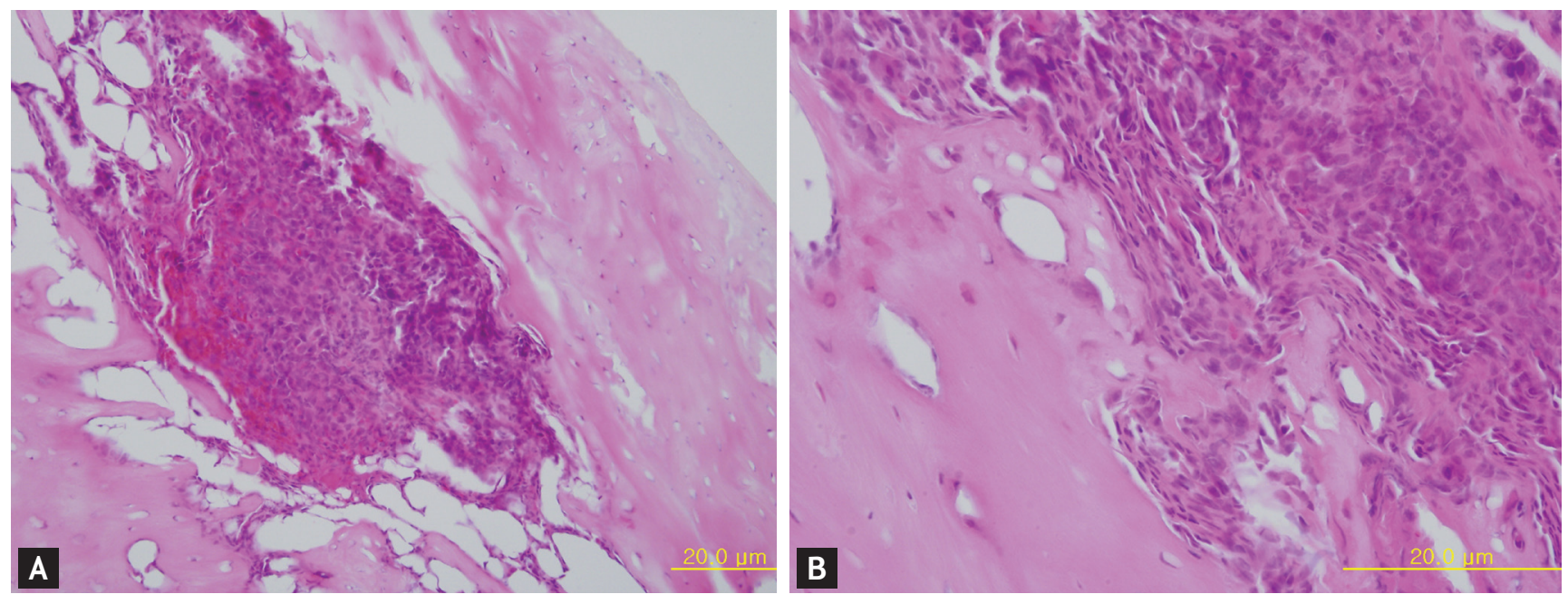

Figure 3. Pathologic findings of the left leg of a cancer-induced bone pain mouse 6 weeks after injection of tumor cells. Many tumor (osteolytic fibrosarcoma) cells have infiltrated and destroyed the bone marrow (A, H\&E, ×200). The tumor cells show highly pleomorphic and prominent nucleoli (B, H\&E, ×400).

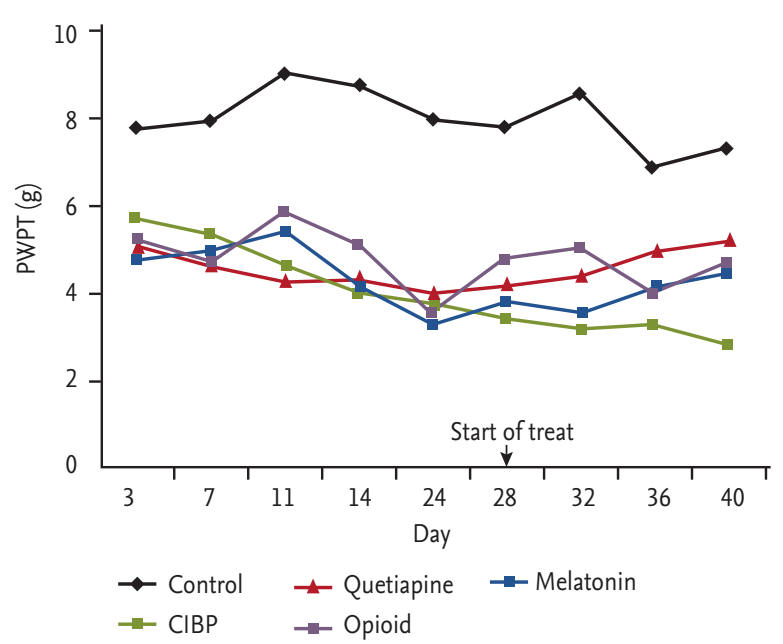

Figure 4. Graph showing the withdrawal pressure threshold of mice in the five groups. The withdrawal pressure threshold is improved in the quetiapine treatment group compared with the cancer-induced bone pain (CIBP) group. PWPT, paw withdrawal pressure threshold.

\section{Expression of acid-sensing ion channels}

The expression levels of TRPV1, TRPV4, ASIC1, ASIC2, and $\mathrm{ASIC}_{3}$ were lower in the CIBP with quetiapine treatment group than in the CIBP group. To investigate the effect of quetiapine on TRPV1, TRPV4, ASIC1, ASIC2, and ASIC3 expression, mRNA levels in the quetiapine treatment group were compared to those in the CIBP with no treatment group and CIBP with fentanyl citrate treatment group. The mRNA levels of TRPV1, TRPV4, ASIC1, ASIC2, and $\mathrm{ASIC}_{3}$ in the CIBP with quetiapine

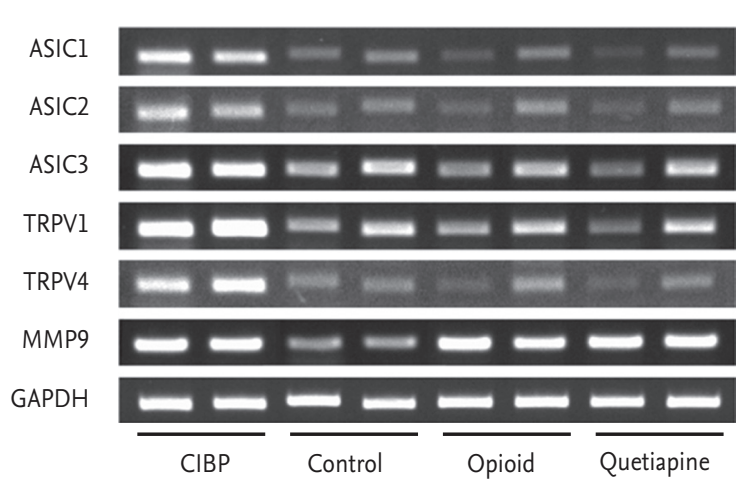

Figure 5. Reverse transcription-polymerase chain reaction analysis of the expression of transient receptor potential vanilloid 1 (TRPV1), TRPV4, acid-sensing ion channel 1 (ASIC1), $\mathrm{ASIC}_{2}$, and $\mathrm{ASIC}_{3}$ in each group. mRNA levels of TRPV1, TRPV4, ASIC1, ASIC2, and ASIC3 are markedly decreased in the quetiapine treatment group comparable to those in the opioid treatment group. $\mathrm{MMP}_{9}$, matrix metallopeptidase 9; GAPDH, glyceraldehyde 3-phosphate dehydrogenase; CIBP, cancer-induced bone pain.

group were markedly decreased and comparable to those in the CIBP with fentanyl citrate group (Fig. 5).

\section{DISCUSSION}

Cancer metastasis to bone results in CIBP and derived from neurochemical changes that are unique compared with other chronic pain states. The acidic tumor environment and secretion of substances such as growth 
factors, cytokines, and chemokines from tumor cells have been reported to stimulate nearby primary afferent nociceptors and induce pain $[16,17]$. The principal challenge in understanding the mechanism of cancer pain is the development of an animal model of pain that displays similar characteristics to human CIBP [18]. The present study demonstrates that fibrosarcoma cell inoculation of the tibia of $\mathrm{C}_{3} \mathrm{H} / \mathrm{HeN}$ mice produces progressive mechanical hyperalgesia, indicating successful establishment of a model of bone pain from metastatic bone cancer. Also, radiologic evaluation of the injected tibia shows progressive bone destruction, which might be the origin of the noxious inputs responsible for the hyperalgesia and allodynia. These findings are in good agreement with the spontaneous and evoked pain in patients with various kinds of bone cancer [19]. The evoked pain behaviors and the response to fentanyl in our experiments are consistent with those observed in other bone cancer models [20].

Quetiapine is an atypical antipsychotic drug and has also been used in the treatment of depression [12]. Numerous studies of the anti-inflammatory effects of antidepressant have been reported [13,14]. The evidence indicates that antidepressants suppress the production of monocytic cytokine, such as interleukin $1 \beta$ and tumor necrosis factor $\alpha$. In 2012, we reported a study of the anti-inflammatory effect of quetiapine on collagen-induced arthritis in a mouse model [15]. That study demonstrated that quetiapine decreased arthritic inflammation and bone destruction in the collagen-induced arthritis mouse model. Quetiapine reduced the severity of arthritis and joint destruction, the underlying mechanism of which may be associated with the inhibitory effect of quetiapine on proinflammatory cytokine production [15].

In the current study, we demonstrated that quetiapine had an analgesic effect in the CIBP animal model by behavior testing. Our data showed that the PWPT was improved in the quetiapine treatment group compared with CIBP group. In addition, we revealed that expression of acid-sensing ion channels was increased in the CIBP animal model and decreased in the quetiapine treatment group and the opioid treatment group. These results raise the possibility that TRPV and ASICs might be potential targets for cancer pain management. However, this experiment had some limitations. First, only three mice were included in each group, for a total of 15 mice; therefore, we could not demonstrate statistical significance. Second, the size of the mice was too small to separate tissue of spinal cord and dorsal root ganglia; therefore, the level of nervous system involved in the analgesic effect of quetiapine was not analyzed, and there was no way to identify structural changes of the spinal cord. Third, we tested only hind paw withdrawal threshold to confirm mechanical allodynia and hyperalgesia. Nonetheless, we successfully generated an animal model of CIBP by injection of tumor cells into the intramedullary space of the mouse tibia. This animal model is available for future expanded studies to reveal the mechanism of cancer pain.

\section{KEY MESSAGE}

1. Quetiapine is an atypical antipsychotic drug, previously it was demonstrated that quetiapine reduced the severity of arthritis and joint destruction by anti-inflammatory effects.

2. This study showed that the mouse behavior and expression of acid-sensing ion channels was improved in the quetiapine treatment group compared with control group in a mouse model.

3. We suggest an analgesic effect of quetiapine in the cancer-induced bone pain animal model and implicate transient receptor potential vanilloid and acid-sensing ion channels as potential targets for cancer pain management.

\section{Conflict of interest}

No potential conflict of interest relevant to this article was reported.

\section{Acknowledgments}

The present research was conducted with funding from a Research Grant of Keimyung University Kidney Institute.

\section{REFERENCES}

1. Goudas LC, Bloch R, Gialeli-Goudas M, Lau J, Carr DB. The epidemiology of cancer pain. Cancer Invest 
2005;23:182-190.

2. Portenoy RK, Lesage P. Management of cancer pain. Lancet 1999;353:1695-1700.

3. Levy MH. Pharmacologic treatment of cancer pain. $\mathrm{N}$ Engl J Med 1996;335:1124-1132.

4. de Wit R, van Dam F, Loonstra S, et al. The Amsterdam Pain Management Index compared to eight frequently used outcome measures to evaluate the adequacy of pain treatment in cancer patients with chronic pain. Pain 2001;91:339-349.

5. Meuser T, Pietruck C, Radbruch L, Stute P, Lehmann KA, Grond S. Symptoms during cancer pain treatment following WHO-guidelines: a longitudinal follow-up study of symptom prevalence, severity and etiology. Pain 2001;93:247-257.

6. Schwei MJ, Honore P, Rogers SD, et al. Neurochemical and cellular reorganization of the spinal cord in a murine model of bone cancer pain. J Neurosci 1999;19:1088610897 .

7. Mantyh PW, Clohisy DR, Koltzenburg M, Hunt SP. Molecular mechanisms of cancer pain. Nat Rev Cancer 2002;2:201-209.

8. Fujii Y, Ozaki N, Taguchi T, Mizumura K, Furukawa K, Sugiura Y. TRP channels and ASICs mediate mechanical hyperalgesia in models of inflammatory muscle pain and delayed onset muscle soreness. Pain 2008;140:292-304.

9. Ikeuchi M, Kolker SJ, Burnes LA, Walder RY, Sluka KA. Role of $\mathrm{ASIC}_{3}$ in the primary and secondary hyperalgesia produced by joint inflammation in mice. Pain 2008;137:662-669.

10. Xu TL, Duan B. Calcium-permeable acid-sensing ion channel in nociceptive plasticity: a new target for pain control. Prog Neurobiol 2009;87:171-180.
11. Julius D, Basbaum AI. Molecular mechanisms of nociception. Nature 2001;413:203-210.

12. Arvanitis LA, Miller BG. Multiple fixed doses of "Seroquel" (quetiapine) in patients with acute exacerbation of schizophrenia: a comparison with haloperidol and placebo: the Seroquel Trial 13 Study Group. Biol Psychiatry 1997;42:233246.

13. Bianchi M, Panerai AE. Antidepressant drugs and experimental inflammation. Pharmacol Res 1996;33:235-238.

14. Taler M, Bar M, Korob I, et al. Evidence for an inhibitory immunomodulatory effect of selected antidepressants on rat splenocytes: possible relevance to depression and hyperactive-immune disorders. Int Immunopharmacol 2008;8:526-533.

15. Kim H, Bang J, Chang HW, et al. Anti-inflammatory effect of quetiapine on collagen-induced arthritis of mouse. Eur J Pharmacol 2012;678:55-60.

16. Lozano-Ondoua AN, Symons-Liguori AM, Vanderah TW. Cancer-induced bone pain: mechanisms and models. Neurosci Lett 2013;557 Pt A:52-59.

17. Watkins LR, Wiertelak EP, Goehler LE, Smith KP, Martin D, Maier SF. Characterization of cytokine-induced hyperalgesia. Brain Res 1994;654:15-26.

18. Jimenez-Andrade JM, Mantyh WG, Bloom AP, Ferng AS, Geffre CP, Mantyh PW. Bone cancer pain. Ann N Y Acad Sci 2010;1198:173-181.

19. Mantyh PW. Cancer pain and its impact on diagnosis, survival and quality of life. Nat Rev Neurosci 2006;7:797809 .

20. Zhang RX, Liu B, Wang L, et al. Spinal glial activation in a new rat model of bone cancer pain produced by prostate cancer cell inoculation of the tibia. Pain 2005;118:125-136. 